\title{
La caricature entre image et parole : Villiers de I'Isle-Adam rival d'Honoré Daumier
}

Le nom d'Honoré Daumier n'est mentionné, dans la correspondance de Villiers de l'Isle-Adam, qu'une seule fois. Mais avec cette remarque, l'écrivain évoque le modèle dont il se réclame afin d'expliquer son projet littéraire. C'est en découvrant la «puissance de grotesque » dont est dotée sa plume que Villiers annonce à Mallarmé sa volonté d'anéantir l'odieux bourgeois :

Le fait est que je ferais du bourgeois, si Dieu me prête vie, ce que Voltaire a fait des 'cléricaux', Rousseau des gentilshommes et Molière des médecins. [...] Enfin nous rirons un peu. On m'a dit que Daumier les flattait servilement en comparaison. Et naturellement, moi, j'ai l'air de les aimer et de les porter aux nues, en les tuant comme des coqs. Vous verrez mes types [...] : je les énamoure et les cisèle avec toute ma complaisance. Bref, je crois que j'ai trouvé le défaut de la cuirasse et que ce sera inattendu. (Villiers de l'Isle-Adam, 1962 [1866], p. 99)

Faisant écho aux grandes voix satiriques de la littérature française, Villiers insiste sur l'importance de son entreprise autant esthétique quethique qui, quoi qu'il en résulte, prêtera à rire tout en prolongeant la tradition classique du comique. Poussé par une fureur de l'idéal et des valeurs spirituelles que la société moderne rejette en faveur de la pensée "positive » et du matérialisme, Villiers de l'Isle-Adam, formule son idée d'une littérature conçue comme une vengeance, ce qui permet d'y voir

Anna Opiela-Mrozik - maître de conférences à l'Institut d'Études romanes de l'Université de Varsovie. Adresse de correspondance : Institut d'Études romanes de l'Université de Varsovie, ul. Dobra 55, 00-312 Varsovie, Pologne; e-mail : am.opiela@uw.edu.pl

ORCID iD : https://orcid.org/0000-0002-4349-0631 
un acte de justice qui se fonde sur une raillerie féroce et dont les ressorts sont mûs par l'ironie, la satire et la caricature. En se servant d'une arme à plusieurs tranchants, Villiers adopte une attitude ambiguë qui relie « la colère bouillante du poète exterminateur et le froid mépris de l'ironiste impassible, le tout mêlé à l'humour pince-sansrire de l'artiste pourfendeur de la stupidité bourgeoise » (Noiray, 2001, p. 55). Jouant sur un rire acerbe et sans indulgence, la caricature villiérienne n'est pas destinée à divertir le public, bien au contraire, son objectif est de dégoûter voire d'effrayer le lecteur par une représentation de la laideur morale.

En adjoignant à ses portraits-charges des caricatures de situation et de langage, Villiers érige la caricature en un nouveau genre littéraire, agissant comme un " art de la transformation " du modèle représenté, c'est-à-dire une pratique fondée sur « l'ambiguité et la condensation» (Kris, 1978, p. 241), une pratique que devrait par ailleurs maitriser tout dessinateur habile. Cet art, comme le suggère Henri Bergson, "qui a quelque chose de diabolique, relève le démon qu'avait terrassé l'ange » (1959, p. 399). Tel paraît être aussi l'objectif de Villiers : dévoiler des déformations dissimulées derrière l'ordre commun, faire découvrir ce qui se cache "sous les harmonies superficielles de la forme » (Bergson, 1959, p. 399). C'est ainsi que, par des éléments textuels apparemment anodins, Villiers transforme les personnages de bourgeois qui, sous les yeux d'un lecteur attentif, se métamorphosent en êtres aussi ridicules que menaçants. Avec les moyens qui lui sont propres, l'écrivain invente un mode d'emploi original de la caricature en transférant sur le terrain de la littérature « la compétition avec les dessinateurs » (Sylvos, 1991, p. 74), et notamment avec Daumier qui, par ailleurs, rejoignait le domaine des lettres au moyen des courtes légendes ajoutées à ses caricatures. C'est pourquoi, il nous paraît intéressant de relever, en nous appuyant sur les représentations de quelques professions et milieux choisis, les traits caractéristiques de la caricature qui se dégagent de la vaste fresque sociale de Daumier, pour ensuite les juxtaposer à la vision défigurée de la réalité proposée par Villiers.

\section{Indulgent comme Daumier?}

Le nombre de lithographies de Daumier est non moins impressionnant que leur caractère totalisant : à travers ces quelques milliers d'images, le dessinateur a réussi à reproduire ce que Balzac représentait dans la Comédie humaine. Même si le nom de Daumier est associé à une satire politique dirigée contre le roi Louis-Philippe, son « œuvre-labyrinthe ", selon l'expression de Baudelaire (1976 II [1868], p. 553), contient toute une galerie de personnages : depuis les êtres illustrant « la friponnerie moderne " (Escholier, 1965, p. 114), ce dont témoigne la série de Robert Macaire placée dans l'univers des bourgeois, jusqu'aux types de misérables de toute espèce, ces héros muets de la vie quotidienne inspirant la pitié plutôt que le rire. 
Mais, quant à sa caricature sensu stricto ${ }^{1}$, abondante en sujets et personnages touchés, il est important de noter qu'elle évolue vers l'atténuation de la portée satirique au profit du développement de l'aspect ludique (voir Guinand, 2015, p. 66). Ayant débuté avec la satire politique, Daumier se tourne ensuite, en raison des lois sur la censure de la presse, vers la caricature de mœurs, convaincu que « le rire est une réponse à l'inquiétude des temps modernes » (Le Men, 2008, p. 128). En essayant de rendre sa propre vision de la société, Daumier oscille entre le réalisme et sa déformation, entre une référence grinçante à la situation politique actuelle et sa représentation fantasque, mais n'hésite pas à tourner la caricature contre son auteur en se caricaturant lui-même, marquant ainsi sa distance face à son art. Il propose une sorte de jeu entre le destinataire de la caricature et le caricaturé, conduisant à l'auto-dérision et au brouillage interprétatif, qui met à mal le récepteur. En effet, la plupart des dessins de Daumier sont pourvus de légendes qui, sous forme de jeux de mots, de propos ironiques ou bien de sous-entendus, complètent le message visuel, sans forcément l'expliciter. Au contraire, c'est dans ce rapport entre le texte et l'image, que réside l'ambiguïté du sens véhiculé par la caricature de Daumier, ce qui l'apparente à l'écriture de Villiers qui laisse le lecteur dans l'incertitude interprétative. Laisser planer le doute sur le véritable objet de la satire est un principe qui s'applique aussi bien à la caricature graphique de Daumier qu'à celle, littéraire, de Villiers ; dans les deux cas, il s'agit d' « une caricature consciente d'elle-même qui, pour préserver la pluralité du réel, se refuse à conclure pour mieux s'élever à la 'blague supérieure' " (Guinand, 2015, p. 175). On assiste ainsi à l'instauration d'un « comique ambigu » (Berthelot, 2008, p. 257) dont le meilleur exemple serait le personnage de Robert Macaire qui, tout en constituant une source du ridicule, se fait critique du principe même de la caricature en redoublant ainsi le sens de celle-ci et augmentant sa portée 2 . Dans une des planches de Daumier (fig. 1), on voit une foule de spectateurs en train de se presser devant la vitrine d'un marchand d'estampes affichant quelques épisodes de Robert Macaire et de son compère Bertrand. Ceux-ci, placés au premier plan du dessin, commentent la scène, en dénonçant les objectifs indignes des caricaturistes qui calomnient la société. Les personnages des caricatures sont donc mobilisés dans cette opération de mise en abîme qui fait preuve du caractère autoréférentiel de la caricature.

1. Certes, la distinction entre la caricature et la satire paraît problématique, les deux termes étant souvent considérés comme interchangeables. Cependant, nous nous proposons de comprendre la caricature en tant que déformation graphique dont l'objectif principal serait la dérision du modèle représenté, ce qui la rapproche de la satire visuelle, tandis que la satire classique, genre fondé sur l'attaque et la raillerie exprimée avec une violence verbale, se fixe un objectif sérieux et éthique : la dénonciation des vices.

2. L'épopée de Robert Macaire, dont le héros éponyme avait été emprunté au mélodrame médiocre de Frédérick Lemaître, a été publiée par Daumier dans Le Charivari (1836-1838). Elle met en scène un personnage qui échappe à tout jugement définitf : ce modèle de fripouille qui incarne le vice présent dans tous les domaines de la vie n'en demeure pas moins un homme sympathique et capable de porter un jugement moral sur la société. 
Figure 1. Daumier, « Je ne sais pas ce qu'on peut trouver d'amusant à toutes ces bêtises là... », Le Charivari, 22 octobre 1837. Les musées de la Ville de Paris.

Robert-Macaire : Je ne sais pas ce qu'on peut trouver d'amusant à toutes ces bêtises là...

Bertrand: Je ne vois pas ce qu'on y trouve de piquant...

Robert-Macaire : C'est dégoûtant ! C'est calomnier la société...

Bertrand : La gendarmerie ne devrait pas souffrir de pareils coquins !...

Robert-Macaire : De qui parlez-vous, imbécile?...

Bertrand : Je parle des caricaturistes...

Robert-Macaire : À la bonne heure!!

La puissance que Daumier attribue à Robert Macaire, type du filou moderne atteint par la fièvre de l'argent, apparait comme une mise en garde contre les ambitions démesurées des bourgeois enrichis et contre les valeurs matérialistes que dénonce également Villiers. Au moyen de l'exagération, le dessinateur dénonce ce qu'il considère comme les principaux vices de la société, et notamment "l'égoïsme, la rapacité, le désordre et, par-dessus tout, l'hypocrisie " (Gaultier, 1906, p. 96), qui se rencontrent chez les politiciens, les gens d'affaires, les hommes de loi et les financiers, autrement dit les professions qu'incarne Robert Macaire.

Une satire sérieuse mais exprimée sous un aspect ludique et pour cela déroutante, tel est l'objectif de Daumier, dont le travail de caricaturiste se fonde sur un sens d'observation fort développé. Dans son Histoire de la caricature moderne, Jules Champfleury dénote dans l'art de Daumier une source de « rire particulier », « un comique novateur et difficile à cerner » (Lo Feudo, 2013, p. 323), dont les valeurs esthétiques résident dans les dessins mêmes, au détriment des légendes dont on pourrait, selon Baudelaire, se passer. À l'instar de Baudelaire, Daumier est à la fois dégoûté et fasciné par l'univers bourgeois de son temps. En sondant ce monde aussi ordinaire qu'excentrique, le dessinateur réussit autant à représenter la laideur, en relevant des imperfections physiques, qu’à mettre en ouvre l'idée baudelairienne du beau

3. Les légendes des caricatures de Daumier étaient écrites par divers auteurs, mais il est certain que le dessinateur lui-même en a fait un grand nombre. Cependant, il avait l'habitude de souligner leur caractère accessoire par rapport à la puissance expressive et le message immédiat du dessin. « La légende », disait-il, « est bien inutile. Si mon dessin ne vous révèle rien, c'est qu'il est mauvais; la légende ne le rendra pas meilleur. S'il est bon, vous le comprenez tout seul » (voir Escholier, 1965, p. 106-108). 
moderne. En tant qu'auteur « d'un des plus magnifiques musées de monstruosités anatomiques qui se puissent concevoir " (la série Les Baigneuses, Escholier, 1965, p. 115), Daumier a relevé dans la réalité parisienne « tout ce qu'elle renferme de trésors effrayants, grotesques, sinistres et bouffons » (Baudelaire, 1976 II [1868], p. 554).

Dans ce précieux défilé de personnages peuplant « une triviale et terrible réalité » (p. 552), certains inspirent pourtant de la sympathie au caricaturiste comme au lecteur, si bien que le dessinateur les traite avec indulgence au lieu de s'en moquer. Les séries des Bons Bourgeois, des Papas ou des Mours conjugales mettent en scène des petits bourgeois, des pères et mères de famille préoccupés par leurs enfants et leurs soucis du quotidien, des êtres vivants et concrets avec leurs qualités et défauts. Aussi, leur caricature engendre-t-elle le comique qui s'appuie sur un curieux mélange d'ironie et d'indulgence en provoquant un rire « sans rancune et sans fiel » (Baudelaire, 1976 II [1868], p. 556), un rire «bien français, tout en finesse et en sous-entendus, rire joyeux et allègre, qui moque sans écorcher, raille sans méchanceté, presque avec intérêt " (Gaultier, 1906, p. 109). C'est précisément ce que les lecteurs du Charivari ou de La Caricature attendent de cet artiste (dont le nom leur échappait souvent), qui, tous les matins, « divertit la population parisienne, [...] satisfait aux besoins de la gaité publique et lui donne sa pâture » (Baudelaire, 1976 II [1868], p. 549). Cette adhésion à la caricature des mours bourgeoises de Daumier relève pourtant d'un phénomène d'identification avec le sujet représenté, étant donné que le caricaturiste vise le même public auquel il s'adresse. La confusion entre le destinataire et l'objet de la satire satisfait l'amour-propre du récepteur et favorise un rire sincère et effréné, assurant le plaisir que recherche le public bourgeois.

\section{L'univers des bourgeois ou une recette de la caricature villiérienne}

Dans l'univers dualiste de Villiers de l'Isle-Adam, où des êtres spirituels sont constamment opposés aux matérialistes bornés, ces derniers fournissent matière à des représentations caricaturales qui s'appuient sur une multiplication de moyens propres aux portraits-charges, et font de lécrivain un maître incomparable de l'exagération et de la démesure. Ceci est bien visible, tout d'abord, dans la création du personnage de Tribulat Bonhomet, le fameux "tueur de cygnes", dont les comportements et les opinions ne sont pas sans rappeler ceux de Robert Macaire, son " cousin germain » en quelque sorte (Berthelot, 2008, p. 255). Bonhomet, qui s'est fait connaître dans Claire Lenoir en tant que scientifique grotesque et inquiétant devient, dans d'autres contes villiériens, un symbole cynique et vulgaire de son siècle matérialiste, à l'instar du héros de Daumier. Cependant, Robert Macaire nous paraît tout d'abord ridicule avant d'être odieux. Il en est autrement de Bonhomet qui, à force de s'imposer dans chaque domaine de la vie, se révèle aussi envahissant que monstrueux. C'est, d'ailleurs, ce que vise Villiers : en présentant avec démesure un bourgeois sot 
et ignoble, il avertit ses lecteurs des dangers de la nature dite bourgeoise, dont personne n'est exempt, pas même l'écrivain : " Je hais le bourgeois, mais le bourgeois dont je parle, j'en trouve autant sous la blouse que sous les noms les plus illustres du monde » (Villiers de l'Isle-Adam, 1986 II, p. 1001).

Malgré tout, une sorte de comique persiste, d'autant plus que les bourgeois villiériens sont une combinaison dêtre humain et d'engin mécanique, ce qui les rend ridicules à la manière de marionnettes qu'on manipule. L'originalité de l'écrivain se définit donc "par le genre particulier de vie qu'il communique à un simple pantin " (Bergson, 1959, p. 401). Telle est Alicia Clary qui se comporte et parle comme si elle était animée par un mécanisme intérieur. Puisque son langage s'appuie sur les répétitions de lieux communs, la « déesse bourgeoise ", dépourvue de toute idée spirituelle, ressemble davantage à un automate que le personnage d'Hadaly, sa version artificielle. C'est la parole complétée du rire qui dégrade Alicia dans les yeux de lord Ewald et d'Edison, en mettant en avant son ridicule. En effet, comme le remarque Pierre Citron, on assiste dans L'Ève future au renversement des choses, car

l'idée à partir de laquelle seulement naît le roman, c'est celle d'une andréide qui, contrairement à tous les êtres artificiels [...], ne soit pas une caricature, inférieure, dérisoire, monstrueuse et plus ou moins démoniaque de son modèle humain : elle lui est infiniment supérieure, non pas seulement en apparence mais en réalité ; elle est un être presque angélique ou une sorte d'idée platonicienne incarnée, et c'est son modèle qui est véritablement sa caricature. (Citron, 1979, p. 17)

Paradoxalement, dans le cas d'Alicia, on a affaire à « l'automatisme installé dans la vie et imitant la vie » (Bergson, 1959, p. 402), tout comme chez un autre bourgeois-automate dans l'univers de Villiers, Félix, le protagoniste du drame La Révolte. Son personnage reste très cohérent tout au long de la pièce : il présente un bourgeois concentré sur ses affaires et incapable de comprendre les motivations de sa femme, même si le départ inattendu de celle-ci bouleverse le rythme habituel de sa vie. L'aspect caricatural du personnage réside d'abord dans son langage corporel, une multitude de gestes et de mouvements qui, dès la première scène, suggèrent un caractère mécanique : Félix « compulse des lettres et des billets de banque » (Villiers de l'Isle-Adam, 1986 I [1870], p. 387), fume un cigare, tend son gilet, se déplace pour ensuite s'allonger, etc. Son comportement exagéré s'intensifie après la sortie d'Élisabeth, lorsqu'il «se frappe le front et s'arrête », « arrache sa cravate » (p. 404), crie et marche avant de s'évanouir.

Mais l'essentiel de la " charge » villiériénne est placée dans le langage de Félix dont le monologue "tend à la caricature ", tandis que celui de sa femme "s'élève vers la poésie » (Raitt et Castex, 1986 I, p. 1139)4. La langue de Félix est celle d'un banquier guidé par le sens commun qui fait de lui un représentant typique de sa classe sociale :

4. La Révolte ne présente pas un vrai dialogue entre les protagonistes, mais une juxtaposition de deux monologues qui s'affrontent. 
un bourgeois positiviste à outrance, privé d'états d'âme et pour cela complètement indifférent aux propos idéalistes d'Élisabeth ${ }^{5}$. Dans le discours de ce monomane " uniquement habité par la logique de l'accumulation et du profit » (Parisse, 2018, p. 87), les termes financiers et commerciaux abondent, même aux moments de la plus haute tension dramatique : l'écart qui se creuse ainsi entre les protagonistes renforce l'effet de caricature anti-bourgeoise. Il suffit de dire que le mari abandonné par sa femme, tout bouleversé qu'il puisse paraître d'après le nombre d'exclamations et autres procédés rhétoriques, conclut son discours de manière à conférer à l'ensemble un statut de caricature précisément ciblée : "Mais il me semble que je me noie, qu'on m’arrache l'existence du corps ! Élisabeth !... Él... (Il fait quelques pas en chancelant, les bras étendus comme un fou, puis il s'affaisse sur un fauteuil auprès de la porte.) Je ne sais pas... mais je souffre beaucoup... positivement " (Villiers de l'Isle-Adam, 1986 I [1870], p. 404). Le discours, en apparence émotionnel, s'achève sur un accent inattendu et grotesque qui ne fait que renforcer le ridicule de ce personnage complètement dominé par l'esprit bourgeois.

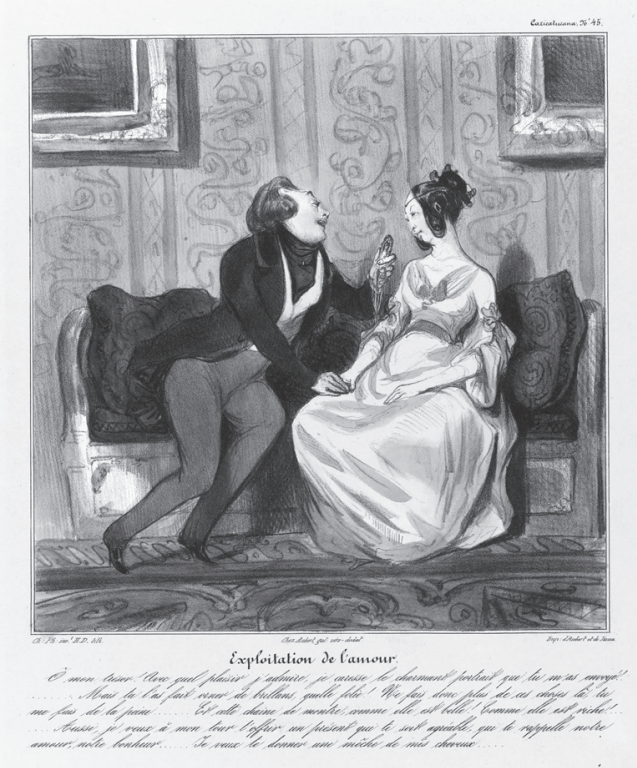

La caricature langagière, qui complète celle de situation, est mise en œuvre, évidemment, dans les Contes cruels où Villiers continue sa mission vengeresse en déployant un talent satirique comparable à celui de Daumier. Évoquons un exemple:parmi les dessins satiriques représentant les bourgeois, on trouve des caricatures de l'avidité ou de l'avarice dans un couple. Le dessin intitulé « Exploitation de l'amour » (fig. 2) montre un amoureux qui tient un discours de convenance face à sa fiancée en feignant de lui reprocher sa « folie» :

Figure 2. Daumier, «Exploitation de l'amour», Le Charivari, 3 mai 1837. Les musées de la Ville de Paris.

Ô mon trésor! Avec quel plaisirj'admire, je caresse le charmant portrait que tu m'as envoyé!........ Mais tu l'as fait orner de brillans, quelle folie! Ne fais donc plus de ces choses là, tu me fais de la peine...... Et cette chaine de montre, comme elle est belle ! Comme elle est riche !..... Aussi, je veux à mon tour t'offrir un présent qui te soit agréable, qui te rappelle notre amour, notre bonheur....... Je veux te donner une mèche de mes cheveux.......

5. Le langage d'Élisabeth relève d'une polyphonie qui empêche d'identifier la psychologie du personnage, contrairement à la cohésion du discours de Félix avec un système de valeurs matérialistes, tantôt celui d'un banquier, tantôt celui d'un philosophe ou bien d'un poète. «Jusqu'à la dernière réplique de la pièce - 'pauvre homme !' - on ne peut tracer un portrait clair d'Élisabeth » (Parisse, 2018, p. 87). 
avoir offert à son bien-aimé un cadeau trop précieux. On peut observer dans cette scène un curieux renversement des rôles : un homme qui offre à une femme une mèche de ses cheveux en récompense d'un portrait et d'un bijou...

Si Daumier se moque d'un futur mari trop radin, le conte de Villiers, Virginie et Paul, va plus loin en mettant en scène un couple d'adolescents, tous deux également obsédés par les questions financières. C’est avec grâce que Villiers mystifie son lecteur en instaurant une ambiance romantique d'une nuit d'avril, propice aux aveux amoureux, afin de l'abolir peu après, lorsque les jeunes fiancés commencent à se parler. Dans cet entretien étonnant où des préoccupations triviales se mêlent aux prétentions poétiques, le mot " argent » revient à chaque réplique et, qui plus est, acquiert un sens ironique dans la description de la nature :

- Paul, Paul, ce n'est pas bien. [...] Elle est vieille et elle nous laissera, aussi, un peu d'argent...

- [...] Mais comme c'est joli, le rossignol! Quelle voix pure et argentine !

- Oui, c'est joli, mais ça empêche de dormir. Il fait très doux, ce soir : la lune est argentée, c'est beau.

- Je savais bien que vous aimiez la poésie, ma cousine.

- Oh ! oui ! la Poésie !... j’étudie le piano. (Villiers de l'Isle-Adam, 1986 I [1883], p. 605)

Même si, selon Pierre Glaudes, Viriginie et Paul « ont conservé la naïveté enfantine de leurs modèles littéraires » $(2015$, p. 128), leur dialogue est à ce point saturé de réflexions matérielles que les deux adolescents se rapprochent plutôt de caricatures de ceux-ci, le récit villiérien se situant d’emblée dans le registre de la parodie du roman Paul et Virginie de Bernardin de Saint-Pierre qui fournit le modèle canonique de l'innocence du premier amour. Chez Villiers, la vraie obsession d'enrichissement, dont témoigne la présence démesurée des mots de la finance à la place de ceux de l'amour, obéit au principe de la charge caricaturale dans la représentation des personnages. Leur " terre-à-terre sans vergogne » (Glaudes, 2015, p. 130) conjuguée à la rhétorique de l'inversion, marquée déjà dans le titre, invite au même rire acerbe quéveillait la planche de Daumier. Avec ces caricatures d’adolescents à la mentalité bourgeoise, Villiers satiriste annonce la désuétude du lyrisme romantique, incompatible avec le siècle positiviste.

\section{La puissance (caricaturale) de la presse}

L'activité du dessinateur et de l'écrivain se déployant dans la presse ${ }^{6}$, celle-ci leur a fourni plusieurs sujets de caricatures. Défendant la presse en tant qu'instrument

6. Villiers fut rédacteur de l'éphémère Revue des Lettres et des Arts (1867-1868) et collaborateur du Gil Blas et du Figaro. Sa carrière littéraire était liée à son activité journalistique étant donné que plusieurs récits réunis ensuite dans Les Contes cruels ont premièrement paru dans la presse. 


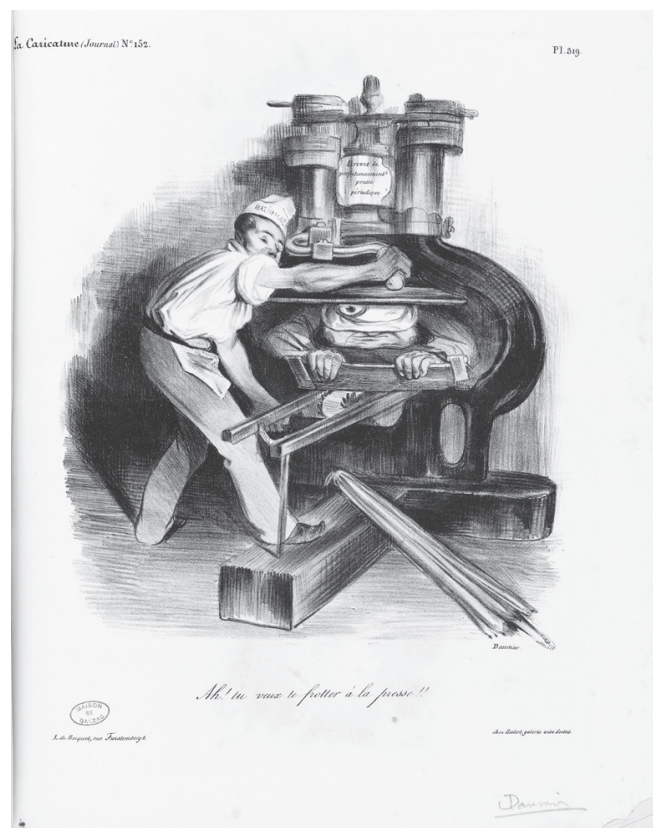

Figure 3. Daumier, «Ah! tu veux te frotter à la presse !! », La Caricature, 3 octobre 1833. Les musées de la Ville de Paris.

de travail et expression de sa liberté, Daumier a créé toute une épopée en images sur les relations entre la presse et le pouvoir ${ }^{7}$. Le dessinateur n'avait pas de doute quant à la puissance des journaux : en jouant sur les mots dans le titre de la caricature « Ah ! tu veux te frotter à la presse !! » (fig. 3), il a averti le roi Louis-Philippe du risque qu'entraînerait son attentat à la liberté de la presse. Le pouvoir dont disposent les directeurs de journaux et qui favorise la corruption, a été bien illustré par Robert Macaire Journaliste qui, guidé par la logique du gain, adresse des propos trompeurs à ses créanciers en les menaçant d'une attaque en diffamation (fig. 4).

Daumier a également relevé l'influence de la presse sur la vie sociale. Aussi, ses caricatures représentent-elles

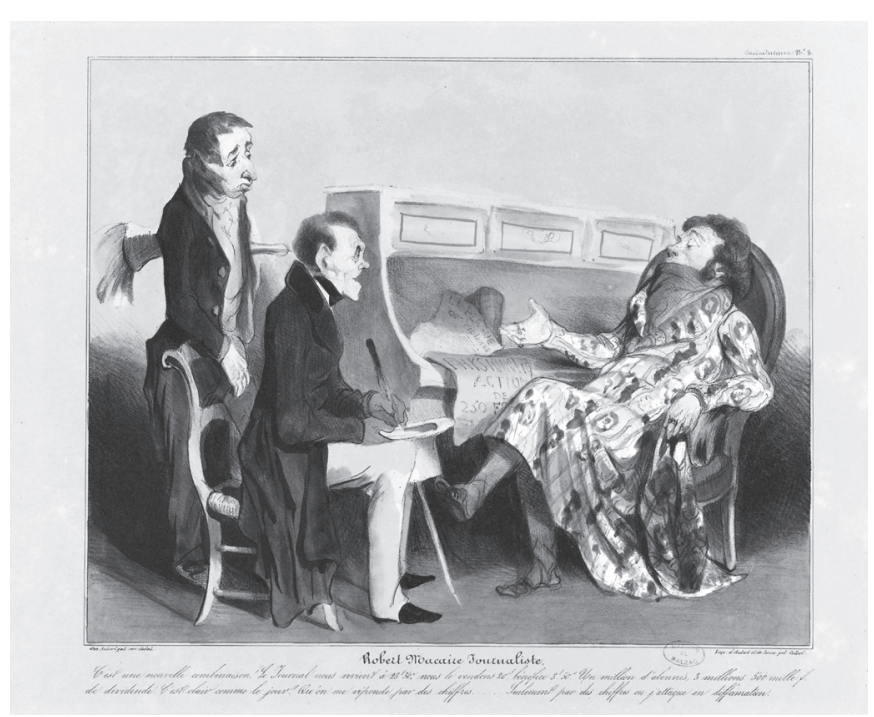

Figure 4. Daumier « Robert Macaire Journaliste », Le Charivari, 10 septembre 1836. Les musées de la Ville de Paris.

C'est une nouvelle combinaison! Le Journal nous revient à $23 \mathrm{f} 50 \mathrm{c}$ nous le vendons $20 \mathrm{f}$ bénéfice $3 \mathrm{f} 50 \mathrm{c}$. Un million d'abonnés, 3 millions 500 mille $f$. de dividende. C'est clair comme le jour ! Qu'on me réponde par des chiffres..... Seulement par des chiffres ou j'attaque en diffamation.

7. Soulignons que l'activité du dessinateur couvre une longue période de la censure sur la presse et sur l'image : depuis la loi de 1835, qui suivit l'attentat de Fieschi contre le roi, en passant par celles de 1849 et de 1850 qui introduisent de nouvelles mesures répressives, jusqu'en 1868 lorsque l'empereur adopte une législation libérale. 
des bourgeois lisant des journaux aux formats monstres dans des situations des plus intimes (à table, en promenade et même au bain). Se remarque ainsi une invasion de la presse à laquelle les bourgeois font confiance, sans se soucier de la véracité des nouvelles qu'on leur fait lire. Par ailleurs, Daumier déplore le foisonnement de nouveaux journaux suite aux lois de 1868, y voyant une " marée " qui entraîne le lecteur, perdu au milieu d'un flux violent d'informations (« La grande marée de 1868 », Le Charivari, 19 décembre 1868).

Les relations de Villiers avec la presse paraissent moins profitables que celles de Daumier. En essayant de se faire publier, Villiers prenait une pose dédaigneuse face aux directeurs des revues, ce dont témoigne un souvenir noté par Remy de Gourmont à propos des rédacteurs du Gil Blas : «Il affectait d'ailleurs devant ces hommes la plus singulière attitude, les accablant de saluts, de compliments, se glissant, en humble collaborateur, heureux d'évoluer parmi tant de maîtres. C'était sa manière de mépriser» (1906, p. 224).

Continuant sa mission de vengeance littéraire, Villiers prend pour cible les hommes de presse dans le conte Deux augures où il met en scène un directeur de journal à qui il oppose un littérateur débutant. S'adressant aux jeunes " âmes de penseurs et d'écrivains " aspirant à conquérir les pages des journaux, Villiers envisage de les désillusionner par la description du " colloque bizarre et d'apparence paradoxale » (Villiers de l'Isle-Adam, 1986 I [1883], p. 566), illustration parfaite du conflit éternel entre le rêve juvénile et la réalité. Le personnage du directeur constitue le pivot central du récit, c'est « un de ces hommes qui traitent les honnêtes bourgeois de 'matière abonnable' " (p. 556), dont le portrait, en apparence normal, se transforme ensuite en caricature de serviteur de la médiocrité bourgeoise. En effet, lors de l'entretien avec un jeune homme de lettres, s'opère un renversement des valeurs (procédé pratiqué aussi par Daumier et porteur d'effet comique) qui dévoile dans le personnage du Journaliste une figure grotesque à la recherche du manque de talents chez l'auteur présumé. Par un geste théâtral, il découvre la supercherie du poète inconnu qui prétendait une absence totale de talent : " Au bout de trois minutes, le directeur tressaille, puis rejette, avec dédain, les feuilles volantes sur la table » en poussant un soupir de déception « Hélas ! mon noble ami, mais c'est plein de talent, ça!» (p. 571).

Suit une longue tirade du directeur dans laquelle les appels au « sérieux » se mêlent aux considérations économiques, le tout servi avec une bonne dose de cynisme insolent, ce qui rend la satire de Villiers d'autant plus poignante. Le directeur du journal, à la manière de ceux que représentait Daumier, est bien conscient de la position supérieure du journalisme, devenu une véritable industrie culturelle, face à la littérature. En faisant répéter au directeur la devise de la médiocrité, mise en exergue de son récit («Surtout, pas de génie!»), Villiers n’a qu'un objectif : «créer un type et illustrer, sous une forme caricaturale, la décadence du goût littéraire moderne, ravalé au plus bas niveau d'un public bourgeois sans goût et sans curiosité intellectuelle » (Raitt et Castex, 1986 I, p. 1272). Même si la réaction du jeune littérateur 
qui passe à l'attaque paraît déroutante ${ }^{8}$, il n'en est pas moins vrai que la caricature villiérienne, plus brutale que ridicule, se veut d'une portée générale en condamnant, encore une fois, la pensée positiviste aveuglément suivie par les bourgeois.

\section{Les artistes qui se font charger}

Dans sa vision de l'univers bourgeois, Daumier a consacré beaucoup de place aux pratiques artistiques populaires au XIX ${ }^{\mathrm{e}}$ siècle. Plusieurs dessins représentent donc des types de musiciens jouant de divers instruments, chanteurs, chefs d'orchestre, acteurs (souvent amateurs), directeurs de théâtre, le public enfin. La vogue de s'adonner à un art d'agrément permet à Daumier de se moquer de ceux qui jouent ou chantent faux ou qui tentent en vain de devenir acteurs. Mais, en même temps, le dessinateur saisit, à travers un miroir déformant, les tendances qu'on peut observer sur les scènes musicales et théâtrales de l'époque : la prédominance de l'instrumentation grandiose, la politique de la recette au théâtre, le déclin de la tragédie classique.

À nouveau, surgit le personnage de Robert Macaire, cette fois en tant que chef d'orchestre. C'est sans doute par allusion à Berlioz qu'on le voit exécuter de la « musique pyrotechnique, charivarique et diabolique » (fig. 5) et, afin de la rendre plus bruyante, qu'il n'hésite pas à compléter l'instrumentation orchestrale des coups de pistolets ou de canon, étant donné que le siècle manque d'harmonie. En revanche, la puissance de la musique de Wagner se laisse quant à elle deviner par les réactions des auditeurs en train d'absorber un tumulte de notes répandu avec violence par l'orchestre lors d'un concert à Munich (« Après une heure de Wagner par ordre !! », Le Charivari, 8 juillet 1868).

Chez Villiers, on trouve également une vision caricaturale du musicien qui, de façon perverse, confirme l'engouement de l'écrivain pour le wagnérisme. C'est dans le conte Le secret de l'ancienne-musique, dédié par ailleurs à Wagner, que l'écrivain excelle et déroute par l'ironie et la caricature. Il met en scène un vieux musicien, le dernier virtuose d'un ancien instrument, le chapeau-chinois, dont la présence parait indispensable pour l'exécution d'une œuvre préparée par l'orchestre de l'Académie nationale de Musique. Rien dans ce récit ne prêterait à la satire si Villiers avait choisi un autre instrument, car celui-ci était en réalité une simple percussion n'exigeant aucune aptitude spéciale pour en sortir un son. C’est sans surprise que le vieux maître

8. En effet, après avoir écouté la tirade du directeur, il s’écrie : «Quoi ! je viens vous offrir une ineptie cent fois inférieure à toutes celles que vous publiez chaque jour, une filandreuse chronique suintant la suffisance repue, le cynisme quiet, la nullité sentencieuse, - l'idéal du genre ! une perle, enfin ! Et voici qu'au lieu de me répondre oui ou non, vous m'accablez d'injures ! [...] Vous me traitez, à brûlepourpoint, de littérateur, d’écrivain, de penseur, que sais-je ? (Villiers de l'Isle-Adam, 1986 I [1883], p. 575). Contrairement aux attentes du lecteur, ayant bien compris les objectifs du directeur du journal, le jeune littérateur ne défend pas les valeurs de l'art véritable, la littérature, mais s'en prend au Journaliste en revendiquant les mêmes valeurs de médiocrité. Il se montre donc non moins cynique que lui. 
Figure 5. Daumier, « Musique pyrotechnique, charivarique et diabolique ", Le Charivari, 11 novembre 1838. Les musées de la Ville de Paris Simple ménétrier de bastringue, Macaire a compris son époque. Nous ne vivons pas dans un temps d'harmonie, il faut du bruit beaucoup de bruit ! c'est pourquoi Macaire fait des vers charabias, introduit les fusées et les pistolets dans la symphonie et fait de la musique à coup de canon... C'est plus ronflant et surtout plus facile !... Habitués des cabarets, cafés borgnas, ... Chefs d'établissements coulés, directeurs de concerts en plein vent, propriétaires de jardins déserts, Macaire serait votre dieu s'il enfonçait Strauss et Musard comme il enfonce ses créanciers. Baouud! Baouud ! Pouff! Pouff!! Pââââouff !!!...

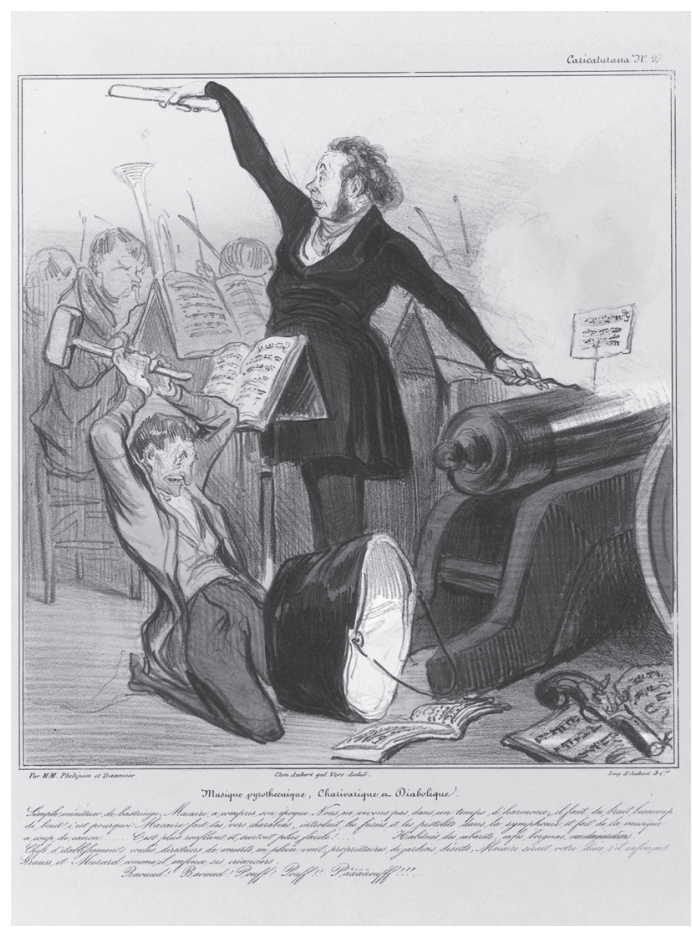

est ridiculisé au moment de l'exécution de la pièce, d'autant plus que sa partition ne se compose que de silences : " Il se roidit à cette vue ; un mouvement fiévreux lui échappa !... Mais rien, dans son instrument, ne trahit les sentiments qui l'agitaient. [...] Il joua. Sans broncher! Avec une maîtrise, une sûreté, un brio, qui frappèrent d'admiration tout l'orchestre " (Villiers de l'Isle-Adam, 1986 I [1883], p. 641).

$\mathrm{La}$ « charge » du portrait du vieillard trouve pourtant son comble après le concert. Afin de le résumer, reprenons le titre d'un dessin de Daumier représentant le musicien : "La grosse caisse interviendra... au dénouement ». C'est ce qui se passe effectivement à la fin du récit, lorsque le musicien de jadis, effrayé par la déchéance de son art auquel manque la mélodie, y renonce à jamais : «Et, foudroyé par son propre transport, il trébucha. Dans sa chute, il creva la grosse caisse et y disparut comme sévanouit une vision! Hélas! il emportait, en s'engouffrant ainsi dans les flancs profonds du monstre, le secret des charmes de l'ancienne-Musique » (p. 641)9.

Qu'en est-il des personnages d'acteurs? Il ne fait pas de doute que Daumier s'intéresse aux tragédiens classiques, ce dont témoignent ses Physionomies tragicoclassiques et ses Physionomies tragiques. La tragédie devient pour lui un prétexte à se

9. Certes, le comique, engendré par une caricature de situation et celle du personnage, n'est pas à nier. Mais le portrait du vieux maître se nuance "d'une certaine sympathie, et même d'un certain pathétique » (Raitt et Castex, 1986, p. 1298) ce qui met le lecteur dans le doute quant aux préférences musicales de Villiers, et rend ambiguë l'interprétation du conte. 
moquer des acteurs qui tentent désespérément de réussir dans ce répertoire exigeant, ainsi que des réactions du public ennuyé par des pièces désuètes. La caricature consiste donc à relever un écart entre l'idéal de l'interprétation et une réalité souvent vulgaire.

Villiers, pour sa part, a fondé sur le personnage de l'acteur un de ses contes les plus originaux intitulé Le Désir d’être un homme. Esprit Chaudval, le héros du récit, est un acteur tragique au seuil de la retraite. Ayant maîtrisé à fond son art et interprété des rôles célèbres, il se rend compte qu'il ne vit pas vraiment, sauf la vie des personnages qu'il a incarnés. Atteint d'une "déformation histrionique » (Pellois, 2014, p. 235), Chaudval apparaît comme une caricature diabolique de l'homme, cet être qu'il désire en vain devenir. Si son monologue prononcé devant un miroir peut encore paraître ridicule, l'acte criminel qui en résultera (l'incendie d'un quartier) est, au contraire, aussi monstrueux qu'inefficace. En cherchant désespérément à ressentir des remords et à voir des spectres, Chaudval se réduit, volontairement, à sa propre caricature, pitoyable et cruelle en même temps, tout comme Alicia Clary reste une version imparfaite et ignoble d'andréïde. Et Villiers de conclure son récit en résumant l'échec de l'artiste déformé par ses illusions : « et le vieux histrion expira, déclamant toujours, en sa vaine emphase, son grand souhait de voir des spectres... » (Villiers de l'Isle-Adam, 1986 I [1883], p. 665). Le portrait de l'acteur, en proie à des enjeux existentiels et victime de sa carrière absurde, paraît bien éloigné des artistes caricaturés par Daumier pour lesquels la scène semble ne relever que du divertissement ou du gain.

\section{Conclusion}

Villiers de l'Isle-Adam a-t-il réussi à dépasser Daumier dans son défi de caricaturiste ambitionnant d'être plus méchant que le dessinateur ? Bien que l'écrivain et le dessinateur choisissent à peu près les mêmes cibles, il est évident que Villiers, tout en s'inspirant de l'œuvre de son prédécesseur, va au-delà de cet héritage graphique. En effet, les objectifs des deux caricaturistes se distinguent. Chez Daumier, la satire violente dirigée contre les hommes politiques coexiste avec un rire joyeux et quasi indulgent qu'engendrent les défauts des bourgeois pour qui le dessinateur garde une forme de tendresse ou de sympathie. Amuser ceux qui en sont l'objet, tel est le but de la caricature graphique de Daumier. Quant à Villiers, dans son œuvre, et notamment dans les Contes cruels, "le rire déclenche la peur » (Sylvos, 1991, p. 79) chez tous ceux qui partagent avec l'écrivain la hantise du matérialisme devenu la nouvelle religion des bourgeois.

La caricature littéraire de Villiers de l'Isle-Adam semble alors s'élever à un autre niveau de la critique, en devenant un instrument qui permet à l'écrivain de mesurer son propre idéalisme. Par ailleurs, dans sa lutte pour celui-ci ne serait-il pas luimême devenu une espèce de caricature d'aristocrate de sang et d'esprit ? Force est 
de rappeler que ce rival de Daumier a été caricaturé en 1890 (un an après sa mort), en couverture de la revue Les Hommes d'aujourd'hui. Villiers y est représenté en tenue de chevalier, armé d'une plume et accoudé sur un tas de ses ouvrages. Voici donc une vie et une œuvre bouclées par la caricature.

\section{RÉFÉRENCES}

Baudelaire, Ch. (1976) [1868]. Quelques caricaturistes français. Dans Euvres complètes, vol. II (p. 544-563). Paris : Gallimard.

Bergson, H. (1959). Le rire. Essai sur la signification du comique. Dans Euvres (p. 381-485). Paris : PUF.

Berthelot, S. (2008). Robert Macaire ou le brouillage satirique. Dans S. Duval et J.-P. Saïdah (dir.), Mauvais genre. La satire littéraire moderne (p. 255-269). Pessac: Presses Universitaires de Bordeaux.

Citron, P. (1979). Introduction à A. Villiers de l'Isle-Adam. L'Ève future (p. 9-26). Lausanne : L'Âge d'homme.

Escholier, R. (1965). Daumier et son monde. Nancy : Éditions Berger-Levrault.

Gaultier, P. (1906). Le rire et la caricature. Paris : Hachette \& Cie.

Glaudes, P. (2015). Cruel retournement de l'idylle. Virginie et Paul de Villiers de l'Isle-Adam. Dans F. Bercegol et H. Mater (dir.), Métamorphoses du roman sentimental. XIX ${ }^{e}-X X I^{e}$ siècle (p. 123-132). Paris : Classiques Garnier.

Gourmont, R. de. (1906). Un carnet de notes sur Villiers de l'Isle-Adam. L'Ermitage, 15 avril 1906, 223-238.

Guinand, C. (2015). La Caricature littéraire : L'Éducation sentimentale de Flaubert. Quêtes littéraires, 5, 65-77. https://doi.org/10.31743/q1.239

Kris, E. et Gombrich, E. H. (1978). Psychanalyse de l'art. Paris : PUF.

Le Men, S. (2008). Daumier et la caricature. Paris : Citadelles \& Mazenod.

Lo Feudo, M. (2013). Penser le rire au XIX ${ }^{\mathrm{e}}$ siècle à travers les Histoires de la caricature. Dans A. Vaillant et R. de Villeneuve (dir.), Le rire moderne (p. 313-333). Nanterre : Presses Universitaires de Paris Nanterre.

Noiray, J. (2001). La littérature comme vengeance : l'esthétique de Villiers de l'Isle-Adam d'après sa correspondance. Dans A. Michel (dir.), L'Esthétique dans les correspondances d'écrivains et de musiciens (XIX'-XXe siècles) (p. 45-56). Paris : PUPS.

Parisse, L. (2018). Portraits féminins dans le théâtre et l'œuvre de Villiers de l'Isle-Adam. Dans J. Anselmini et F. Bercegol (dir.), Portraits dans la littérature. De Gustave Flaubert à Marcel Proust (p. 81-103). Paris : Classiques Garnier.

Pellois, A. (2014). Modèles de l'acteur chez Villiers de l'Isle-Adam : du 'spectre' à 'l'identité idéalisée'. Littératures, 71, 229-242.

Raitt, A. et Castex, P.-G. (1986). Notices des Contes cruels. Dans A. Villiers de l'Isle-Adam, Euvres complètes (p. 1261-1343). Paris : Gallimard.

Sylvos, F. (1991). L'essence cruelle du rire : Villiers de l'Isle-Adam. Romantisme, 74, 73-82.

Villiers de l'Isle-Adam, A. (1962). Correspondance générale de Villiers de l'Isle-Adam. vol. I. J. Bollery (éd.). Paris : Mercure de France.

Villiers de l'Isle-Adam, A. (1986). Euvres complètes, vol. I et II. Paris : Gallimard. 


\section{FIGURES}

Daumier, H. (22 octobre 1839). Je ne sais pas ce qu'on peut trouver d'amusant à toutes ces bêtises là. Le Charivari. Les musées de la Ville de Paris.

https://www.parismuseescollections.paris.fr/fr/maison-de-balzac/oeuvres/robert-macaireje-ne-sais-pas-ce-qu-on-peut-trouver-d-amusant-a-toutes-ces

Daumier, H. (3 mai 1837). Exploitation de l'amour. Le Charivari. Les musées de la Ville de Paris.

https://www.parismuseescollections.paris.fr/fr/maison-de-balzac/oeuvres/exploitation-de-l-amour

Daumier, H. (3 octobre 1833). Ah ! tu veux te frotter à la presse !!. La Caricature. Les musées de la Ville de Paris.

https://www.parismuseescollections.paris.fr/fr/maison-de-balzac/oeuvres/ah-tu-veux-tefrotter-a-la-presse

Daumier, H. (10 septembre 1836). Robert Macaire Journaliste, Le Charivari. Les musées de la Ville de Paris.

https://www.parismuseescollections.paris.fr/fr/maison-de-balzac/oeuvres/robert-macaire-journaliste

Daumier, H. (11 novembre 1838). Musique pyrotechnique, charivarique et diabolique. Le Charivari. Les musées de la Ville de Paris.

https://www.parismuseescollections.paris.fr/fr/maison-de-balzac/oeuvres/musique-pyrothecnique-charivarique-et-diabolique

RÉSUMÉ : L'article propose d'analyser, dans une optique comparatiste, les objectifs et les spécificités de la caricature graphique d'Honoré Daumier et de la caricature littéraire de Villiers de l'Isle-Adam. Par son projet de vengeance satirique sur les bourgeois, Villiers de l'Isle-Adam se réclame de la méthode de Daumier, fondée sur l'ironie, et envisage de dépasser le dessinateur. Dans la présentation de l'univers bourgeois et des milieux choisis (journalistique, artistique) le dessinateur et l'écrivain élaborent leurs propres façons d'exploiter les ressources de la caricature. Tout en dénonçant les vices de la société, Daumier propose un type particulier de comique (dans lequel la légende joue un rôle secondaire) et développe l'aspect ludique de son art, tandis que Villiers se concentre sur la raillerie violente, même si sa caricature relève souvent de l'ambiguïté. Érigée en genre littéraire, la caricature villiérienne relie le portrait déformé à la caricature langagière et celle de situation, en élargissant ainsi les sens véhiculés par le dessin de Daumier.

Mots-clés : caricature, Daumier, Villiers de l’Isle-Adam, satire, bourgeoisie 
The caricature between the image and the word: Villiers de I'Isle-Adam as a rival of Honoré Daumier

ABSTRACT: This article analyzes, from a comparative perspective, the aims and the characteristics of two types of caricature: Honoré Daumier's graphic caricature and Villiers de l'Isle-Adam's literary one. For the purposes of his satiric revenge on bourgeoisie, Villiers resorted, by means of irony, to Daumier's method, even trying to overshadow him. In their way of presenting the world of bourgeoisie and selected social groups (journalist and artistic ones), this draughtsman and this writer worked out their own means of the use of caricature. Without stopping stigmatising the vices of society, Daumier proposed a peculiar kind of caricature (in which an explanation played a secondary role) and developed the ludic aspect of his art, whereas Villiers was focused on mockery, even though his caricatures were often ambiguous. Villiers' caricature, which even became a literary genre, connects a distorted portrait with a linguistic and situational caricature, by which means it makes the sense, formed by Daumier's image, broader.

Keywords: caricature, Daumier, Villiers de l'Isle-Adam, satire, bourgeoisie 